\title{
Synthesis of 2-(3-methyl-2-oxoquinoxalin-1(2H)- yl)acetamide Based Thiazolidinone Derivatives as Potent Antibacterial and Antifungal Agents
}

\author{
SHIV KUMAR ${ }^{\mathrm{a}^{*}}$, NITIN KUMAR ${ }^{\mathrm{b}}$, SUSHMA DRABU ${ }^{\mathrm{c}}$, SUROOR AHMAD KHAN ${ }^{\mathrm{d}}$, \\ OZAIR ALAM $^{\mathrm{d}}$, MANAV MALHOTRA $^{\mathrm{e}}$, AND MD. AKRAM MINHAJ ${ }^{\mathrm{a}}$
}

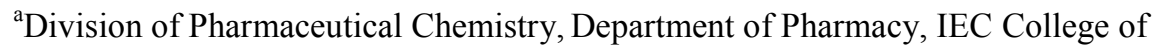

Engineering \& Technology, Knowledge Park-I, Greater Noida-201306 (India)

${ }^{b}$ Department of Pharmaceutical Technology, Meerut Institute of Engineering \& Technology, Meerut-250005, India

${ }^{c}$ Maharaja Surajmal Institute of Pharmacy, Janak Puri, New Delhi-110058, India ${ }^{\mathrm{d}}$ Department of Pharmaceutical Chemistry, Faculty of Pharmacy, Jamia Hamdard (Hamdard University), Hamdard Nagar, New Delhi-110062, India

${ }^{\mathrm{e}}$ Department of Pharmaceutical Chemistry, ISF College of Pharmacy, Ferozpur Road, Moga142001, India

shiv_hamdard@yahoo.co.in

shiv_hamdard@rediffmail.com

Received 17 November 2011; Accepted 15 January 2012

\begin{abstract}
Twelve compounds belonging to series 2-(3-methyl-2oxoquinoxalin-1(2H)-yl)- $N$-[4-oxo-2-(substituted)phenyl-1,3-thiazolidin-3yl]acetamide (5a-l) were synthesized. These compounds were evaluated for their in-vitro antibacterial against E. coli, S. aureus, K. pneumoniae, P.aeruginosa \& antifungal activity against $C$. albicans, A. niger \& A. flavus by cup-plate method. Structures of all the newly synthesized compounds were confirmed by elemental analysis, ${ }^{1}$ H-NMR \& FT-IR spectral data interpretation. Compounds $5 \mathbf{d} \& \mathbf{5 h}$ having $p$-nitrophenyl \& $p$ trifluoromethylphenyl group respectively on 2-position of thiazolidinone ring attached to N-atom of acetamido group on 1-position of 3-methyl-1Hquinoxaline-2-one, were found to be active against all the bacterial \& fungal strains under investigation, while compound $\mathbf{5}$ having $p$-chlorophenyl on 2 position of thiazolidinone nucleus was reported as least active compound against all bacterial \& fungal strain under investigation.
\end{abstract}

Keywords: Quinoxaline, Thiazolidinone, Antibacterial, Antifungal.

\section{Introduction}

Nitrogen containing heterocyclic compounds are indispensable structural units for both the chemists \& biochemists. Among the various classes of benzene fused six-membered 
nitrogen containing heterocyclic compounds, quinoxaline derivatives form important classes of pharmacologically active compounds. Quinoxaline ring is a part of various antibiotics such as hinomycin, levomycin, and actinoleutin that are known to inhibit growth of gram positive bacteria and are active against various transplantable tumors. ${ }^{1-7}$ Quinoxaline derivatives have also been found to be associated with a wide variety of biological activities including antifungal ${ }^{8-10}$, antibacterial ${ }^{10-14}$, antitubercular ${ }^{8,9,15-18}$, anti-inflammatory agents ${ }^{19}$. Further hydrazinoquinoxalines \& their cyclic analogues have been reported as antimicrobial agents $^{20}$. Five member nitrogen \& sulphur containing thiazolidinone ring attached with other heterocyclic system have also been found to be associated with wide spectrum of pharmacological activities viz. antibacterial ${ }^{21,22,25,26}$, antifungal ${ }^{22}$, anti-inflammatory ${ }^{23}$, antitumor $^{24}$, anti-HIV ${ }^{25}$, etc.

Keeping the above facts in view, it was thought worthwhile to design the synthesis of compound series viz. 2-(3-methyl-2-oxoquinoxalin-1(2H)-yl)- $N$-[4-oxo-2(substituted)phenyl-1,3-thiazolidin-3-yl]acetamide (5a-1) wherein thiazolidinone rings have been attached to quinoxaline at 1 position with an acetamido amino linkage. All the synthesized have been screened for in-vitro antibacterial activity against gram positive bacteria Staphylococcus aureus (ATCC2913), gram negative bacteria Klebsella pneumoniae (ATCC700603), Pseudomonas aeruginosa (ATCC27853), Escherichia coli (ATCC25922) $\&$ antifungal activity against Candida albicans (MTCC3017), Aspergillus niger (MTCC281) and Aspergillus flavus (MTCC222) by using cup-plate method.

\section{Experimental}

\section{General}

Melting points were determined in open capillary tubes in a Hicon melting point apparatus and are uncorrected. All the Fourier Transform-Infra Red (FT-IR) spectra were recorded in $\mathrm{KBr}$ pellets on a Shimadzu-8400S spectrometer. The ${ }^{1} \mathrm{H}-\mathrm{NMR}$ spectra were taken on a Bruker-Spectrospin DCX (300 MHz) NMR spectrometer. Chemical shifts $(\delta)$ are expressed in ppm relative to tetramethylsilane (TMS), used as an internal standard. The purity of the compounds was checked by thin layer chromatography (TLC) on Merck Silica Gel $60_{\mathrm{F} 254}$ precoated sheets. Iodine chamber and UV lamp were used for the visualization of TLC spots.

\section{General procedure for the synthesis of titled compounds}

3-Methyl-1H-quinoxaline-2-one (1): Pyruvic acid $(0.1 \mathrm{~mol})$ and $o$-phenylenediamine $(0.1 \mathrm{~mol})$, in $20 \% \mathrm{HCl}(100 \mathrm{ml})$, were mixed properly and heated at $45^{\circ} \mathrm{C}$ with continuous stirring for about 5 hours. Product was filtered, dried and washed with water, dried and then purified by dissolving in $5 \% \mathrm{w} / \mathrm{v} \mathrm{NaOH}(75 \mathrm{ml})$. Then, liquid was filtered and cooled to temperature below $5^{\circ} \mathrm{C}$ and acidified with glacial acetic acid to $\mathrm{pH}$ 6. Buff colored crystals appeared \& then recrystallized with dimethylformamide (DMF). Pale yellow crystals, yield $60 \%, \mathrm{R}_{f \text { (Ethylacetate : Hexane }=7: 3)} 0.73$, m.p. $230^{\circ} \mathrm{C},{ }^{1} \mathrm{H}-\mathrm{NMR}\left(\mathrm{DMSO}-d_{6}, 300 \mathrm{MHz}, \delta \mathrm{ppm}\right)$ : 7.624-7.651 (d, $1 \mathrm{H}$, quinoxaline ring protons, $\mathrm{J}=8.1 \mathrm{~Hz}), 7.391-7.441(\mathrm{t}, 1 \mathrm{H}$, quinoxaline ring protons, $\mathrm{J}=5.4-9.6 \mathrm{~Hz}), 7.188-7.250(\mathrm{~m}, 2 \mathrm{H}$, quinoxaline ring protons, $\mathrm{J}=2.7-7.5 \mathrm{~Hz})$, $2.364\left(\mathrm{~s}, 3 \mathrm{H}, \mathrm{CH}_{3}\right), 9.1(\mathrm{~s}, 1 \mathrm{H}, \mathrm{NHCO})$; IR $\left(\mathrm{KBr}, \mathrm{cm}^{-1}\right)$ : 762 (ortho-disubstituted aromatic ring), $1390(\mathrm{C}-\mathrm{N}), 1500$ (aromatic $\mathrm{C}=\mathrm{C}), 1544(-\mathrm{NH}), 1688(>\mathrm{C}=\mathrm{O}), 3314(-\mathrm{NH})$.

Ethyl-[3-methyl-2-oxo-quinoxaline-1-yl]acetate (2): Compound (1) (0.0642 mol), ethylchloroacetate $(0.077 \mathrm{~mol})$ and potassium carbonate $(0.078 \mathrm{~mol})$ were taken in round bottom flask and refluxed in acetone $(100 \mathrm{ml})$ for 6 hours. After completion of reaction, acetone was removed by reduced pressure. The residue was added to ice cold water (300 
$\mathrm{ml}$ ), acidified with acetic acid, filtered, washed with cold water, dried and finally recrystallized with ethylacetate. Pale yellow crystals, yield $45 \%, \mathrm{R}_{f \text { (Ethylacetate }: \text { Hexane }=7: 3)} 0.64$, m.p. $112^{\circ} \mathrm{C},{ }^{1} \mathrm{H}-\mathrm{NMR}\left(\mathrm{DMSO}-d_{6}, 300 \mathrm{MHz}, \delta \mathrm{ppm}\right): 7.739-7.766(\mathrm{~d}, 1 \mathrm{H}$, quinoxaline ring protons, $\mathrm{J}=8.1 \mathrm{~Hz}), 7.532-7.559(\mathrm{t}, 1 \mathrm{H}$, quinoxaline ring protons, $\mathrm{J}=3.9-4.2 \mathrm{~Hz}), 7.436-$ $7.46(\mathrm{~d}, 1 \mathrm{H}$, quinoxaline ring protons, $\mathrm{J}=3 \mathrm{~Hz}), 7.314-7.364(\mathrm{t}, 1 \mathrm{H}$, quinoxaline ring protons, $\mathrm{J}=6.6-8.4 \mathrm{~Hz}$ ), 4.118-4.189 (q, 2H, $\left.\mathrm{CH}_{2} \mathrm{OCO}\right), 3.386\left(\mathrm{~s}, 2 \mathrm{H}, \mathrm{CH}_{2} \mathrm{CO}\right), 2.397$ (s, $\left.3 \mathrm{H}, \mathrm{CH}_{3}\right), 1.149-1.196\left(\mathrm{t}, 3 \mathrm{H}, \mathrm{CH}_{3}\right)$; IR $\left(\mathrm{KBr}, \mathrm{cm}^{-1}\right)$ : 763 (ortho-disubstituted aromatic ring), $962($ aromatic $=\mathrm{C}-\mathrm{H}$, bend $), 1199(=\mathrm{C}-\mathrm{N}), 1285(\mathrm{C}-\mathrm{O}$, coupled with H-C-H$), 1380(\mathrm{C}-\mathrm{N})$, $1645(\mathrm{CH}=\mathrm{N}), 1750(>\mathrm{C}=\mathrm{O}), 2745(\mathrm{C}-\mathrm{C})$.

3-Methyl-2-oxo-quinoxaline-1(2H)-acetohydrazide (3): Compound $2(0.02 \mathrm{~mol})$ and hydrazine hydrate $(0.03 \mathrm{~mol})$ were dissolved in ethanol $(50 \mathrm{ml})$ and refluxed for 6 hours. Reaction mixture was kept in deep freezer for overnight. Product was filtered, dried and finally recrystallized with DMF. Biege colored solid, yield $50 \%, \mathrm{R}_{f \text { (TEF) }} 0.9$, m.p. $200{ }^{\circ} \mathrm{C},{ }^{1} \mathrm{H}$ NMR (DMSO- $\left.d_{6}, 300 \mathrm{MHz}, \delta \mathrm{ppm}\right): 7.729-7.755(\mathrm{~d}, 1 \mathrm{H}$, quinoxaline ring protons, $\mathrm{J}=7.8$ $\mathrm{Hz})$, 7.494-7.545 (t, $1 \mathrm{H}$, quinoxaline ring protons, $\mathrm{J}=6.3-9 \mathrm{~Hz}), 7.262-7.354(\mathrm{q}, 2 \mathrm{H}$, quinoxaline ring protons, $\mathrm{J}=7.5-10.2 \mathrm{~Hz}), 4.843\left(\mathrm{~s}, 2 \mathrm{H}, \mathrm{NHNH}_{2}\right.$, exchangeable protons), 4.284 (s, $\left.2 \mathrm{H}, \mathrm{CH}_{2} \mathrm{CO}\right), 2.432\left(\mathrm{~s}, 3 \mathrm{H}, \mathrm{CH}_{3}\right)$; IR $\left(\mathrm{KBr}, \mathrm{cm}^{-1}\right)$ : 760 (ortho-disubstituted aromatic ring), 963 (aromatic $=\mathrm{C}-\mathrm{H}$, bend), $1198(=\mathrm{C}-\mathrm{N}), 1385(\mathrm{C}-\mathrm{N}), 1569(-\mathrm{NH}$, bend), 1645 $(\mathrm{CH}=\mathrm{N}), 1749(>\mathrm{C}=\mathrm{O}), 2320(\mathrm{~N}-\mathrm{N}), 3245(-\mathrm{NH}$. str.).

2-(3-Methyl-2-oxoquinoxalin-1(2H)-yl)- $N^{\prime}$-[(substituted)phenylmethylidene]aceto hydrazide (4a-l): Equimolar quantities of compound 3 (0.0044 mol) and aromatic aldehydes $(0.0045 \mathrm{~mol})$ were dissolved in methanol $(25 \mathrm{ml})$ and refluxed for 6 hours. A solid appears with vigorous bumping in each case. Solid was filtered, washed with cold methanol, dried and recrystallized with DMF.

N'-benzylidene-2-(3-methyl-2-oxoquinoxalin-1(2H)-yl)acetohydrazide (4a): Light yellow solid, yield $54 \%, \mathrm{R}_{f \text { (Benzene : Acetone }=8: 2)} 0.85$, m.p. $234^{\circ} \mathrm{C},{ }^{1} \mathrm{H}-\mathrm{NMR}$ (DMSO- $d_{6}, 300$ $\mathrm{MHz}, \delta \mathrm{ppm}): 8.061(\mathrm{~s}, 1 \mathrm{H}, \mathrm{NHCO}), 7.678-7.776(\mathrm{~m}, 4 \mathrm{H}$, quinoxaline ring protons, $\mathrm{J}=3-$ $7.5 \mathrm{~Hz}), 7.418-7.433(\mathrm{~m}, 5 \mathrm{H}, \mathrm{Ar}-\mathrm{H}, \mathrm{J}=2.7-8.1 \mathrm{~Hz}), 5.425\left(\mathrm{~s}, 2 \mathrm{H}, \mathrm{CH}_{2}\right), 5.035(\mathrm{~s}, 1 \mathrm{H}$, $\mathrm{CH}=\mathrm{N}), 2.545\left(\mathrm{~s}, 3 \mathrm{H}, \mathrm{CH}_{3}\right)$; IR $\left(\mathrm{KBr}, \mathrm{cm}^{-1}\right): 723$ (mono-substituted aromatic ring), 769 (ortho-disubstituted aromatic ring), 854 (aromatic $\mathrm{C}=\mathrm{C}$ ), 964 (aromatic $=\mathrm{C}-\mathrm{H}$, bend), 1199 $(=\mathrm{C}-\mathrm{N}) 1388(\mathrm{C}-\mathrm{N}), 1542(-\mathrm{NH}$, bend $), 1650(\mathrm{CH}=\mathrm{N}), 1650(\mathrm{CH}=\mathrm{N}), 1729(>\mathrm{C}=\mathrm{O}), 2345$ $(\mathrm{N}-\mathrm{N}=), 3244(-\mathrm{NH})$.

N'-(4-chlorobenzylidene)-2-(3-methyl-2-oxoquinoxalin-1(2H)-yl)acetohydrazide

(4b): Light yellow solid, yield $48 \%, \mathrm{R}_{f}$ (Benzene : Acetone $=8: 2$ ) 0.72 , m.p. $230^{\circ} \mathrm{C},{ }^{1} \mathrm{H}-\mathrm{NMR}$ $\left(\mathrm{DMSO}_{6}, 300 \mathrm{MHz}, \delta \mathrm{ppm}\right): 8.032-8.067$ (q, $4 \mathrm{H}, \mathrm{A}_{2} \mathrm{~B}_{2}$ pattern, $\mathrm{Ar}-\mathrm{H}, \mathrm{J}=3.3-3.6 \mathrm{~Hz}$ ), $8.188(\mathrm{~s}, 1 \mathrm{H}, \mathrm{CONH}), 7.578-7.768(\mathrm{~m}, 4 \mathrm{H}$, quinoxaline ring protons, $\mathrm{J}=2.7-8.1 \mathrm{~Hz}), 5.425$ $\left(\mathrm{s}, 2 \mathrm{H}, \mathrm{CH}_{2}\right), 5.036(\mathrm{~s}, 1 \mathrm{H}, \mathrm{CH}=\mathrm{N}), 2.354\left(\mathrm{~s}, 3 \mathrm{H}, \mathrm{CH}_{3}\right) ; \mathrm{IR}\left(\mathrm{KBr}, \mathrm{cm}^{-1}\right): 765$ (orthodisubstituted aromatic ring), 823 (para-disubstituted aromatic ring), 865 (aromatic $\mathrm{C}=\mathrm{C}$ ), 965 (aromatic $=\mathrm{C}-\mathrm{H}$, bend), 1129 (Aryl C-Cl), $1203(=\mathrm{C}-\mathrm{N}), 1385(\mathrm{C}-\mathrm{N}), 1540(-\mathrm{NH}$, bend), $1650(\mathrm{CH}=\mathrm{N}), 1650(\mathrm{CH}=\mathrm{N}), 1740(>\mathrm{C}=\mathrm{O}), 2342(\mathrm{~N}-\mathrm{N}=), 3245(-\mathrm{NH})$.

N'-(4-hydroxybenzylidene)-2-(3-methyl-2-oxoquinoxalin-1(2H)-yl)acetohydrazide (4c): Greenish yellow solid, yield $45 \%, \mathrm{R}_{f}$ (Benzene : Acetone $\left.=8: 2\right) 0.76$, m.p. $254^{\circ} \mathrm{C},{ }^{1} \mathrm{H}-\mathrm{NMR}$ $\left(\mathrm{DMSO}-d_{6}, 300 \mathrm{MHz}, \delta \mathrm{ppm}\right)$ : 8.028-8.062 (q, 4H, A $\mathrm{A}_{2} \mathrm{~B}_{2}$ pattern, Ar-H, J = 3-3.6 Hz), 8.152 $(\mathrm{s}, 1 \mathrm{H}, \mathrm{CONH}), 7.758-7.876(\mathrm{~m}, 4 \mathrm{H}$, quinoxaline ring protons, $2.1-8.4 \mathrm{~Hz}), 5.325(\mathrm{~s}, 2 \mathrm{H}$, $\left.\mathrm{CH}_{2}\right), 5.135(\mathrm{~s}, 1 \mathrm{H}, \mathrm{CH}=\mathrm{N}), 2.486(\mathrm{~s}, 1 \mathrm{H}, \mathrm{OH}), 2.354\left(\mathrm{~s}, 3 \mathrm{H}, \mathrm{CH}_{3}\right) ; \mathrm{IR}\left(\mathrm{KBr}, \mathrm{cm}^{-1}\right): 764$ (ortho-disubstituted aromatic ring), 825 (para-disubstituted aromatic ring), 863 (aromatic $\mathrm{C}=\mathrm{C}), 960$ (aromatic =C-H, bend), $1198(=\mathrm{C}-\mathrm{N}), 1288(\mathrm{C}-\mathrm{O}$, coupled with $\mathrm{H}-\mathrm{C}-\mathrm{H}), 1382$ $(\mathrm{C}-\mathrm{N}), 1540(-\mathrm{NH}$, bend $), 1650(\mathrm{CH}=\mathrm{N}), 1650(\mathrm{CH}=\mathrm{N}), 1745(>\mathrm{C}=\mathrm{O}), 2344(\mathrm{~N}-\mathrm{N}=), 3250$ (-NH), 3675 (phenolic -OH). 


\section{2-(3-methyl-2-oxoquinoxalin-1(2H)-yl)-N'-(4-nitrobenzylidene)acetohydrazide}

(4d): Grey solid, yield $52 \%, \mathrm{R}_{f}$ (Benzene : Acetone $\left.=8: 2\right)$ 0.70, m.p. $220^{\circ} \mathrm{C},{ }^{1} \mathrm{H}-\mathrm{NMR}$ (DMSO- $d_{6}$, $300 \mathrm{MHz}, \delta \mathrm{ppm}): 8.033-064\left(\mathrm{q}, 4 \mathrm{H}, \mathrm{A}_{2} \mathrm{~B}_{2}\right.$ pattern, Ar-H, J = 3-3.3 Hz), $8.154(\mathrm{~s}, 1 \mathrm{H}$, $\mathrm{CONH}), 7.778-7.876(\mathrm{~m}, 4 \mathrm{H}$, quinoxaline ring protons, $\mathrm{J}=2.4-7.5 \mathrm{~Hz}), 5.525\left(\mathrm{~s}, 2 \mathrm{H}, \mathrm{CH}_{2}\right)$, $5.033(\mathrm{~s}, 1 \mathrm{H}, \mathrm{CH}=\mathrm{N}), 2.543\left(\mathrm{~s}, 3 \mathrm{H}, \mathrm{CH}_{3}\right)$; IR $\left(\mathrm{KBr}, \mathrm{cm}^{-1}\right): 723$ (ortho-disubstituted aromatic ring), 829 (para-disubstituted aromatic ring), 864 (aromatic $\mathrm{C}=\mathrm{C}$ ), 969 (aromatic $=\mathrm{C}-\mathrm{H}$, bend), 1199 (=C-N), 1369 (aromatic $-\mathrm{NO}_{2}$ sym. str.), $1385(\mathrm{C}-\mathrm{N}), 1542$ (-NH, bend), 1551 (aromatic $-\mathrm{NO}_{2}$, asym. str.) $1648(\mathrm{CH}=\mathrm{N}), 1748(>\mathrm{C}=\mathrm{O}), 2341(\mathrm{~N}-\mathrm{N}=), 3247(-\mathrm{NH})$.

$\mathrm{N}^{\prime}$-(4-methoxybenzylidene)-2-(3-methyl-2-oxoquinoxalin-1(2H)-yl)acetohydrazide (4e): Light yellow solid, yield $43 \%, \mathrm{R}_{f}$ (Benzene : Acetone $\left.=8: 2\right) 0.80$, m.p. $218^{\circ} \mathrm{C},{ }^{1} \mathrm{H}-\mathrm{NMR}$ $\left(\mathrm{DMSO}_{6}, 300 \mathrm{MHz}, \delta \mathrm{ppm}\right): 8.020-8.052(\mathrm{~d}, 4 \mathrm{H}, \mathrm{Ar}-\mathrm{H}, \mathrm{J}=9.6 \mathrm{~Hz}), 8.142(\mathrm{~s}, 1 \mathrm{H}$, $\mathrm{CONH}), 7.578-7.678(\mathrm{~m}, 4 \mathrm{H}$, quinoxaline ring protons, $\mathrm{J}=2.4-7.5 \mathrm{~Hz}), 5.525\left(\mathrm{~s}, 2 \mathrm{H}, \mathrm{CH}_{2}\right)$, $5.024(\mathrm{~s}, 1 \mathrm{H}, \mathrm{CH}=\mathrm{N}), 3.369\left(\mathrm{~s}, 3 \mathrm{H}, \mathrm{OCH}_{3}\right), 2.351\left(\mathrm{~s}, 3 \mathrm{H}, \mathrm{CH}_{3}\right)$; IR $\left(\mathrm{KBr}, \mathrm{cm}^{-1}\right)$ : 769 (orthodisubstituted aromatic ring), 825 (para-disubstituted aromatic ring), 865 (aromatic $\mathrm{C}=\mathrm{C}$ ), 964 (aromatic $=\mathrm{C}-\mathrm{H}$, bend), $1040(\mathrm{C}-\mathrm{O}-\mathrm{C}$, sym. str.), 1129 (aromatic C-O), $1198(=\mathrm{C}-\mathrm{N})$, 1240 (C-O-C, asym. str.), 1388 (C-N), 1540 (-NH, bend), $1648(\mathrm{CH}=\mathrm{N}), 1745(>\mathrm{C}=\mathrm{O})$, $2344(\mathrm{~N}-\mathrm{N}=), 3250(-\mathrm{NH})$.

$\mathrm{N}^{\prime}$-(3,4-dimethoxybenzylidene)-2-(3-methyl-2-oxoquinoxalin-1(2H)-yl)aceto

hydrazide (4f): Light yellow solid, yield $40 \%, \mathrm{R}_{f}$ (Benzene : Acetone $\left.=8: 2\right) 0.72$, m.p. $208^{\circ} \mathrm{C},{ }^{1} \mathrm{H}$ NMR (DMSO- $\left.d_{6}, 300 \mathrm{MHz}, \delta \mathrm{ppm}\right): 8.112(\mathrm{~s}, 1 \mathrm{H}, \mathrm{CONH}), 7.812-7.998(\mathrm{~m}, 4 \mathrm{H}$, quinoxaline ring protons, $\mathrm{J}=2.4-7.5 \mathrm{~Hz}), 7.775-7.796(\mathrm{~d}, 1 \mathrm{H}, \mathrm{Ar}-\mathrm{H}, \mathrm{J}=6.3 \mathrm{~Hz}), 7.672-$ $7.694(\mathrm{~d}, 1 \mathrm{H}, \mathrm{Ar}-\mathrm{H}, \mathrm{J}=6.6 \mathrm{~Hz}), 7.625(\mathrm{~s}, 1 \mathrm{H}, \mathrm{Ar}-\mathrm{H}), 5.335\left(\mathrm{~s}, 2 \mathrm{H}, \mathrm{CH}_{2}\right), 5.025(\mathrm{~s}, 1 \mathrm{H}$, $\mathrm{CH}=\mathrm{N}), 3.624\left(\mathrm{~s}, 6 \mathrm{H}, \mathrm{OCH}_{3}\right), 2.454\left(\mathrm{~s}, 3 \mathrm{H}, \mathrm{CH}_{3}\right)$; IR $\left(\mathrm{KBr}, \mathrm{cm}^{-1}\right): 768$ (ortho-disubstituted aromatic ring), 830 (para-disubstituted aromatic ring), 863 (aromatic $\mathrm{C}=\mathrm{C}$ ), 965 (aromatic =C-H, bend), 1042 (C-O-C, sym. str.), 1132 (aromatic C-O), $1199(=\mathrm{C}-\mathrm{N}), 1245(\mathrm{C}-\mathrm{O}-\mathrm{C}$, asym. str.), $1385(\mathrm{C}-\mathrm{N}), 1545(-\mathrm{NH}$, bend $), 1650(\mathrm{CH}=\mathrm{N}), 1745(>\mathrm{C}=\mathrm{O}), 2344(\mathrm{~N}-\mathrm{N}=), 3245$ $(-\mathrm{NH})$.

$\mathrm{N}^{\prime}$-(4-(dimethylamino)benzylidene)-2-(3-methyl-2-oxoquinoxalin-1(2H)-

yl)acetohydrazide (4g): Yellow solid, yield $54 \%, \mathrm{R}_{f}$ (Benzene : Acetone $\left.=8: 2\right) 0.86$, m.p. $228^{\circ} \mathrm{C}$, ${ }^{1} \mathrm{H}-\mathrm{NMR}$ (DMSO- $d_{6}, 300 \mathrm{MHz}, \delta \mathrm{ppm}$ ) : 7.033-7.080 (q, 4H, $\mathrm{A}_{2} \mathrm{~B}_{2}$ pattern, Ar-H, J = $3-5.7$ $\mathrm{Hz}), 7.812(\mathrm{~s}, 1 \mathrm{H}, \mathrm{CONH}), 7.558-7.678(\mathrm{~m}, 4 \mathrm{H}$, quinoxaline ring protons, $\mathrm{J}=2.1-8.1 \mathrm{~Hz})$, $5.225\left(\mathrm{~s}, 2 \mathrm{H}, \mathrm{CH}_{2}\right), 5.015(\mathrm{~s}, 1 \mathrm{H}, \mathrm{CH}=\mathrm{N}), 2.353\left(\mathrm{~s}, 3 \mathrm{H}, \mathrm{CH}_{3}\right), 1.832\left(\mathrm{~s}, 6 \mathrm{H}, \mathrm{N}-\mathrm{CH}_{3}\right) ; \mathrm{IR}$ $\left(\mathrm{KBr}, \mathrm{cm}^{-1}\right)$ : 769 (ortho-disubstituted aromatic ring), 830 (para-disubstituted aromatic ring), 865 (aromatic $\mathrm{C}=\mathrm{C}), 968$ (aromatic $=\mathrm{C}-\mathrm{H}$, bend), $1199(=\mathrm{C}-\mathrm{N}), 1384(\mathrm{C}-\mathrm{N}), 1550(-\mathrm{NH}$, bend), $1648(\mathrm{CH}=\mathrm{N}), 1750(>\mathrm{C}=\mathrm{O}), 2344(\mathrm{~N}-\mathrm{N}=), 3124\left(-\mathrm{CH}_{3}\right), 3245(-\mathrm{NH})$.

2-(3-methyl-2-oxoquinoxalin-1(2H)-yl)-N'-(4-(trifluoromethyl)benzylidene)

acetohydrazide (4h): Light yellow solid, yield $46 \%, \mathrm{R}_{f(\text { Benzene }: \text { Acetone }=8: 2)} 0.85$, m.p. $238^{\circ} \mathrm{C}$, ${ }^{1} \mathrm{H}-\mathrm{NMR}$ (DMSO- $\left.d_{6}, 300 \mathrm{MHz}, \delta \mathrm{ppm}\right): 8.184(\mathrm{~s}, 1 \mathrm{H}, \mathrm{CONH}), 7.868-8.072(\mathrm{~m}, 4 \mathrm{H}$, quinoxaline ring protons, $\mathrm{J}=1.8-7.5 \mathrm{~Hz}$ ), 8.133-8.177 (q, $4 \mathrm{H}, \mathrm{A}_{2} \mathrm{~B}_{2}$ pattern, $\mathrm{Ar}-\mathrm{H}, \mathrm{J}=3-5.1$ $\mathrm{Hz}), 5.325\left(\mathrm{~s}, 2 \mathrm{H}, \mathrm{CH}_{2}\right), 5.136(\mathrm{~s}, 1 \mathrm{H}, \mathrm{CH}=\mathrm{N}), 2.645\left(\mathrm{~s}, 3 \mathrm{H}, \mathrm{CH}_{3}\right) ; \mathrm{IR}\left(\mathrm{KBr}, \mathrm{cm}^{-1}\right): 768$ (ortho-disubstituted aromatic ring), 845 (para-disubstituted aromatic ring), 869 (aromatic $\mathrm{C}=\mathrm{C}), 968$ (aromatic =C-H, bend), $1199(=\mathrm{C}-\mathrm{N}), 1288(\operatorname{aryl~C}-\mathrm{F}), 1385(\mathrm{C}-\mathrm{N}), 1548(-\mathrm{NH}$, bend), $1650(\mathrm{CH}=\mathrm{N}), 1745(>\mathrm{C}=\mathrm{O}), 2344(\mathrm{~N}-\mathrm{N}=), 3245(-\mathrm{NH})$.

$\mathrm{N}^{\prime}$-(2-hydroxybenzylidene)-2-(3-methyl-2-oxoquinoxalin-1(2H)-yl)acetohydrazide (4i): Light yellow solid, yield $48 \%, \mathrm{R}_{f}$ (Benzene : Acetone $=8: 2$ ) 0.80 , m.p. $248^{\circ} \mathrm{C},{ }^{1} \mathrm{H}-\mathrm{NMR}$ $\left(\mathrm{DMSO}-d_{6}, 300 \mathrm{MHz}, \delta \mathrm{ppm}\right): 8.152(\mathrm{~s}, 1 \mathrm{H}, \mathrm{CONH}), 7.778-7.876(\mathrm{~m}, 4 \mathrm{H}$, quinoxaline ring protons, , $\mathrm{J}=1.8-6.9 \mathrm{~Hz}), 7.522-7.538(\mathrm{~d}, 1 \mathrm{H}, \mathrm{Ar}-\mathrm{H}, \mathrm{J}=4.8 \mathrm{~Hz}), 7.333-7.359$ (d, 1H, Ar-H, $\mathrm{J}=7.8 \mathrm{~Hz}), 7.251-7.288(\mathrm{t}, 2 \mathrm{H}, \mathrm{Ar}-\mathrm{H}, \mathrm{J}=4.8-5.7 \mathrm{~Hz}), 5.525\left(\mathrm{~s}, 2 \mathrm{H}, \mathrm{CH}_{2}\right), 5.036(\mathrm{~s}, 1 \mathrm{H}$, $\mathrm{CH}=\mathrm{N}), 2.486(\mathrm{~s}, 1 \mathrm{H}, \mathrm{OH}), 2.545\left(\mathrm{~s}, 3 \mathrm{H}, \mathrm{CH}_{3}\right) ; \mathrm{IR}\left(\mathrm{KBr}, \mathrm{cm}^{-1}\right): 765$ (ortho-disubstituted aromatic ring), 868 (aromatic $\mathrm{C}=\mathrm{C}), 969$ (aromatic $=\mathrm{C}-\mathrm{H}$, bend), $1198(=\mathrm{C}-\mathrm{N}), 1258(\mathrm{C}-\mathrm{O}$, 
str.) $1385(\mathrm{C}-\mathrm{N}), 1545(-\mathrm{NH}$, bend), $1649(\mathrm{CH}=\mathrm{N}), 1748(>\mathrm{C}=\mathrm{O}), 2340(\mathrm{~N}-\mathrm{N}=), 3250(-$ $\mathrm{NH}), 3365$ (phenolic $-\mathrm{OH})$.

N'-(3-hydroxy-4-methoxybenzylidene)-2-(3-methyl-2-oxoquinoxalin-1(2H)yl)acetohydrazide $(4 \mathrm{j})$ : Yellow solid, yield $66 \%, \mathrm{R}_{f(\text { Benzene }: \text { Acetone }=8: 2)} 0.87$, m.p. $188^{\circ} \mathrm{C},{ }^{1} \mathrm{H}$ NMR (DMSO- $\left.d_{6}, 300 \mathrm{MHz}, \delta \mathrm{ppm}\right): 8.122(\mathrm{~s}, 1 \mathrm{H}, \mathrm{CONH}), 7.878-7.978(\mathrm{~m}, 4 \mathrm{H}$, quinoxaline ring protons, $\mathrm{J}=2.1-8.4 \mathrm{~Hz}), 7.634(\mathrm{~s}, 1 \mathrm{H}, \mathrm{Ar}-\mathrm{H}), 7.775-7.812(\mathrm{t}, 1 \mathrm{H}, \mathrm{Ar}-\mathrm{H}, \mathrm{J}$ $=3-8.2 \mathrm{~Hz}), 7.824-7.846(\mathrm{~d}, 1 \mathrm{H}, \mathrm{Ar}-\mathrm{H}, \mathrm{J}=6.6 \mathrm{~Hz}), 5.225\left(\mathrm{~s}, 2 \mathrm{H}, \mathrm{CH}_{2}\right), 5.125(\mathrm{~s}, 1 \mathrm{H}$, $\mathrm{CH}=\mathrm{N}), 2.485(\mathrm{~s}, 1 \mathrm{H}, \mathrm{OH}), 2.545\left(\mathrm{~s}, 3 \mathrm{H}, \mathrm{CH}_{3}\right), 3.625\left(\mathrm{~s}, 3 \mathrm{H}, \mathrm{OCH}_{3}\right) ; \mathrm{IR}\left(\mathrm{KBr}, \mathrm{cm}^{-1}\right): 769$ (ortho-disubstituted aromatic ring), 849 (para-disubstituted aromatic ring), 869 (aromatic $\mathrm{C}=\mathrm{C}), 968$ (aromatic $=\mathrm{C}-\mathrm{H}$, bend), 1045 (C-O-C, sym. str.), 1132 (aromatic C-O), 1199 (=C-N), 1245 (C-O-C, asym. str.), 1252 (C-O, str.), 1384 (C-N), 1546 (-NH, bend), 1648 $(\mathrm{CH}=\mathrm{N}), 1749(>\mathrm{C}=\mathrm{O}), 2345(\mathrm{~N}-\mathrm{N}=), 3245(-\mathrm{NH}), 3352$ (phenolic $-\mathrm{OH})$.

N'-(2-fluorobenzylidene)-2-(3-methyl-2-oxoquinoxalin-1(2H)-yl)acetohydrazide

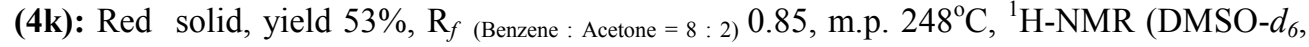
$300 \mathrm{MHz}, \delta \mathrm{ppm}): 8.148(\mathrm{~s}, 1 \mathrm{H}, \mathrm{CONH}), 7.881-7.986(\mathrm{~m}, 4 \mathrm{H}$, quinoxaline ring protons, $\mathrm{J}=$ 1.8-7.5 Hz), 7.623-7.651 (d, 1H, Ar-H, J = 8.4 Hz), 7.436-7.461 (d, 1H, Ar-H, J = 7.5 Hz), 7.551-7.592 (t, 2H, Ar-H, J = 2.4-5.1 Hz), $5.136\left(\mathrm{~s}, 2 \mathrm{H}, \mathrm{CH}_{2}\right), 5.235(\mathrm{~s}, 1 \mathrm{H}, \mathrm{CH}=\mathrm{N}), 2.552$ (s, $3 \mathrm{H}, \mathrm{CH}_{3}$ ); IR $\left(\mathrm{KBr}, \mathrm{cm}^{-1}\right)$ : 769 (ortho-disubstituted aromatic ring), 864 (aromatic $\mathrm{C}=\mathrm{C}$ ), 968 (aromatic $=\mathrm{C}-\mathrm{H}$, bend), $1199(=\mathrm{C}-\mathrm{N}), 1285($ aryl C-F $), 1387(\mathrm{C}-\mathrm{N}), 1548(-\mathrm{NH}$, bend $)$, $1650(\mathrm{CH}=\mathrm{N}), 1745(>\mathrm{C}=\mathrm{O}), 2345(\mathrm{~N}-\mathrm{N}=), 3248(-\mathrm{NH})$.

N'-(2-chlorobenzylidene)-2-(3-methyl-2-oxoquinoxalin-1(2H)-yl)acetohydrazide

(4l): Light yellow solid, yield $43 \%, \mathrm{R}_{f}$ (Benzene : Acetone $\left.=8: 2\right) \quad 0.80$, m.p. $218^{\circ} \mathrm{C},{ }^{1} \mathrm{H}-\mathrm{NMR}$ $\left(\mathrm{DMSO}-d_{6}, 300 \mathrm{MHz}, \delta \mathrm{ppm}\right): 8.136(\mathrm{~s}, 1 \mathrm{H}, \mathrm{CONH}), 7.782-7.880(\mathrm{~m}, 4 \mathrm{H}$, quinoxaline ring protons, , $\mathrm{J}=1.8-7.8 \mathrm{~Hz}), 7.523-7.556(\mathrm{~d}, 1 \mathrm{H}, \mathrm{Ar}-\mathrm{H}, \mathrm{J}=9.9 \mathrm{~Hz}), 7.336-7.362(\mathrm{~d}, 1 \mathrm{H}, \mathrm{Ar}-\mathrm{H}$, $\mathrm{J}=7.8 \mathrm{~Hz}), 7.452-7.496(\mathrm{t}, 2 \mathrm{H}, \mathrm{Ar}-\mathrm{H}, \mathrm{J}=6.6 \mathrm{~Hz}), 5.038\left(\mathrm{~s}, 2 \mathrm{H}, \mathrm{CH}_{2}\right), 5.136(\mathrm{~s}, 1 \mathrm{H}, \mathrm{CH}=\mathrm{N})$, $2.548\left(\mathrm{~s}, 3 \mathrm{H}, \mathrm{CH}_{3}\right)$; IR $\left(\mathrm{KBr}, \mathrm{cm}^{-1}\right)$ : 768 (ortho-disubstituted aromatic ring), 865 (aromatic $\mathrm{C}=\mathrm{C}), 968$ (aromatic $=\mathrm{C}-\mathrm{H}$, bend), $1106(\operatorname{aryl} \mathrm{C}-\mathrm{Cl}), 1198(=\mathrm{C}-\mathrm{N}), 1385(\mathrm{C}-\mathrm{N}), 1545(-\mathrm{NH}$, bend), $1648(\mathrm{CH}=\mathrm{N}), 1752(>\mathrm{C}=\mathrm{O}), 2346(\mathrm{~N}-\mathrm{N}=), 3245(-\mathrm{NH})$.

2-(3-methyl-2-oxoquinoxalin-1(2H)-yl)- $N$-[4-oxo-2-(substituted)phenyl-1,3-

thiazolidin-3-yl]acetamide (5a-l): Compound (4a-1) $(100 \mathrm{mg})$ and thioglycolic acid (10 ml) were refluxed on heavy petroleum ether bath at $200^{\circ} \mathrm{C}$ for 48 hours. Excess of thioglycolic acid was removed by vacuum distillation. Reaction mixture was neutralized with $10 \%$ sodium carbonate solution. Precipitate thus obtained was filtered, dried and recrystallized with DMF.

2-(3-methyl-2-oxoquinoxalin-1(2H)-yl)- $\mathrm{N}$-(4-oxo-2-phenyl-1,3-thiazolidin-3yl)acetamide (5a): Brown solid, yield $48 \%, \mathrm{R}_{f \text { (TEF) }} 0.80$, m.p. $200^{\circ} \mathrm{C},{ }^{1} \mathrm{H}-\mathrm{NMR}$ (DMSO- $d_{6}$, $300 \mathrm{MHz}, \delta \mathrm{ppm}): 8.124(\mathrm{~s}, 1 \mathrm{H}, \mathrm{NHCO}), 7.716-7.798(\mathrm{~m}, 4 \mathrm{H}$, quinoxaline ring protons, $\mathrm{J}=$ 1.8-7.2 Hz), 7.515-7.599(m, 5H, Ar-H, J = 1.5-7.5 Hz), $5.406\left(\mathrm{~s}, 2 \mathrm{H}, \mathrm{CH}_{2}\right), 2.320(\mathrm{~s}, 3 \mathrm{H}$, $\mathrm{CH}_{3}$ ), 3.745 (s, 1H, CH-Ar), $2.512\left(\mathrm{~s}, 2 \mathrm{H}, \mathrm{CH}_{2}-\mathrm{S}\right)$; IR $\left(\mathrm{KBr}, \mathrm{cm}^{-1}\right)$ : 726 (mono-substituted aromatic ring), 762 (ortho-disubstituted aromatic ring), 799 (C-S, str.), 856 (aromatic $\mathrm{C}=\mathrm{C}$ ), $962($ aromatic $=\mathrm{C}-\mathrm{H}$, bend), $1199(=\mathrm{C}-\mathrm{N}), 1385(\mathrm{C}-\mathrm{N}), 1545(-\mathrm{NH}$, bend $), 1745(>\mathrm{C}=\mathrm{O})$, $3245(-\mathrm{NH})$.

$N$-[2-(4-chlorophenyl)-4-oxo-1,3-thiazolidin-3-yl]-2-(3-methyl-2-oxoquinoxalin1(2H)-yl)acetamide (5b): Brown solid, yield $51 \%, \mathrm{R}_{f}$ (TEF) 0.81 , m.p. $212^{\circ} \mathrm{C},{ }^{1} \mathrm{H}-\mathrm{NMR}$ (DMSO- $\left.d_{6}, 300 \mathrm{MHz}, \delta \mathrm{ppm}\right): 8.118(\mathrm{~s}, 1 \mathrm{H}, \mathrm{NHCO}), 7.712-7.794(\mathrm{~m}, 4 \mathrm{H}$, quinoxaline ring protons, $\mathrm{J}=1.8-7.8 \mathrm{~Hz}), 7.527-7.581\left(\mathrm{q}, 4 \mathrm{H}, \mathrm{A}_{2} \mathrm{~B}_{2}\right.$ pattern, Ar- $\left.\mathrm{H}, \mathrm{J}=3-5.1 \mathrm{~Hz}\right), 5.410(\mathrm{~s}$, $\left.2 \mathrm{H}, \mathrm{CH}_{2}\right), 2.311\left(\mathrm{~s}, 3 \mathrm{H}, \mathrm{CH}_{3}\right), 3.788$ (s, $\left.1 \mathrm{H}, \mathrm{CH}-\mathrm{Ar}\right), 2.518$ (s, 2H, $\left.\mathrm{CH}_{2}-\mathrm{S}\right)$; IR $\left(\mathrm{KBr}, \mathrm{cm}^{-1}\right)$ : 762 (ortho-disubstituted aromatic ring), 799 (C-S, str.), 837 (para-disustituted aromatic ring), 852 (aromatic $\mathrm{C}=\mathrm{C}), 966$ (aromatic $=\mathrm{C}-\mathrm{H}$, bend), 1041 (aryl C-Cl,str.), $1198(=\mathrm{C}-\mathrm{N})$, 1388 (C-N), 1549 (-NH, bend), $1748(>\mathrm{C}=\mathrm{O}), 3225(-\mathrm{NH})$. 
$\mathrm{N}$-[2-(4-hydroxyphenyl)-4-oxo-1,3-thiazolidin-3-yl]-2-(3-methyl-2-oxoquinoxalin1(2H)-yl)acetamide (5c): Brown solid, yield $45 \%$, $\mathrm{R}_{f}$ (TEF) 0.78 , m.p. $215^{\circ} \mathrm{C},{ }^{1} \mathrm{H}-\mathrm{NMR}$ $\left(\right.$ DMSO- $\left.d_{6}, 300 \mathrm{MHz}, \delta \mathrm{ppm}\right): 8.128(\mathrm{~s}, 1 \mathrm{H}, \mathrm{NHCO}), 7.718-7.795(\mathrm{~m}, 4 \mathrm{H}$, quinoxaline ring protons, $\mathrm{J}=1.5-7.2 \mathrm{~Hz}), 7.427-7.494(\mathrm{~m}, 4 \mathrm{H}, \mathrm{Ar}-\mathrm{H}, \mathrm{J}=2.1-6.9 \mathrm{~Hz}), 5.418\left(\mathrm{~s}, 2 \mathrm{H}, \mathrm{CH}_{2}\right)$, $2.308\left(\mathrm{~s}, 3 \mathrm{H}, \mathrm{CH}_{3}\right), 3.772(\mathrm{~s}, 1 \mathrm{H}, \mathrm{CH}-\mathrm{Ar}), 2.516\left(\mathrm{~s}, 2 \mathrm{H}, \mathrm{CH}_{2}-\mathrm{S}\right), 3.261$ (s, 1H, phenolic $\mathrm{OH})$; IR $\left(\mathrm{KBr}, \mathrm{cm}^{-1}\right)$ : 765 (ortho-disubstituted aromatic ring), 795 (C-S, str.), 838 (paradisustituted aromatic ring), 851 (aromatic $\mathrm{C}=\mathrm{C}), 969($ aromatic $=\mathrm{C}-\mathrm{H}$, bend), $1199(=\mathrm{C}-\mathrm{N})$, 1257 (C-O, str.), $1385(\mathrm{C}-\mathrm{N}), 1555$ (-NH, bend), $1732(>\mathrm{C}=\mathrm{O}), 3240(-\mathrm{NH}), 3362$ (phenolic $-\mathrm{OH}, \mathrm{str}$.$) .$

2-(3-methyl-2-oxoquinoxalin-1(2H)-yl)- $\mathrm{N}$-[2-(4-nitrophenyl)-4-oxo-1,3-thiazolidin3-yl]acetamide (5d): Brown solid, yield $45 \%, \mathrm{R}_{f(\mathrm{TEF})} 0.78$, m.p. $215^{\circ} \mathrm{C},{ }^{1} \mathrm{H}-\mathrm{NMR}$ (DMSO$\left.d_{6}, 300 \mathrm{MHz}, \delta \mathrm{ppm}\right): 8.132(\mathrm{~s}, 1 \mathrm{H}, \mathrm{NHCO}), 7.715-7.799(\mathrm{~m}, 4 \mathrm{H}$, quinoxaline ring protons, $\mathrm{J}=1.5-6.9 \mathrm{~Hz}), 7.456-7.489\left(\mathrm{q}, 4 \mathrm{H}, \mathrm{A}_{2} \mathrm{~B}_{2}\right.$ pattern, Ar-H, $\left.\mathrm{J}=3-3.9 \mathrm{~Hz}\right), 5.409\left(\mathrm{~s}, 2 \mathrm{H}, \mathrm{CH}_{2}\right)$, 2.308 (s, 3H, $\left.\mathrm{CH}_{3}\right), 3.781$ (s, 1H, CH-Ar), 2.532 (s, 2H, $\mathrm{CH}_{2}-\mathrm{S}$ ); IR $\left(\mathrm{KBr}, \mathrm{cm}^{-1}\right)$ : 769 (orthodisubstituted aromatic ring), 798 (C-S, str.), 835 (para-disustituted aromatic ring), 854 $($ aromatic $\mathrm{C}=\mathrm{C}), 965$ (aromatic $=\mathrm{C}-\mathrm{H}$, bend), $1197(=\mathrm{C}-\mathrm{N}), 1342\left(\right.$ sym . aromatic $\left.-\mathrm{NO}_{2}\right)$, 1388 (C-N), 1518 (asym. aromatic $-\mathrm{NO}_{2}$, str.), 1542 (-NH, bend), $1749(>\mathrm{C}=\mathrm{O}), 3245$ ($\mathrm{NH})$.

$\mathrm{N}$-[2-(4-methoxyphenyl)-4-oxo-1,3-thiazolidin-3-yl]-2-(3-methyl-2-oxoquinoxalin1(2H)-yl)acetamide (5e): Brown solid, yield $62 \%, \mathrm{R}_{f}$ (TEF) 0.80 , m.p. $223^{\circ} \mathrm{C},{ }^{1} \mathrm{H}-\mathrm{NMR}$ (DMSO- $\left.d_{6}, 300 \mathrm{MHz}, \delta \mathrm{ppm}\right): 8.124(\mathrm{~s}, 1 \mathrm{H}, \mathrm{NHCO}), 7.710-7.792(\mathrm{~m}, 4 \mathrm{H}$, quinoxaline ring protons, $\mathrm{J}=1.5-7.2 \mathrm{~Hz}), 7.426-7.488(\mathrm{~m}, 4 \mathrm{H}, \mathrm{Ar}-\mathrm{H}, \mathrm{J}=3-5.1 \mathrm{~Hz}), 5.411\left(\mathrm{~s}, 2 \mathrm{H}, \mathrm{CH}_{2}\right), 2.298$ (s, $\left.3 \mathrm{H}, \mathrm{CH}_{3}\right), 3.755$ (s, $\left.1 \mathrm{H}, \mathrm{CH}-\mathrm{Ar}\right), 2.518$ (s, 2H, $\left.\mathrm{CH}_{2}-\mathrm{S}\right), 2.380$ (s, 3H, $\left.-\mathrm{OCH}_{3}\right)$; IR ( $\mathrm{KBr}$, $\mathrm{cm}^{-1}$ ): 764 (ortho-disubstituted aromatic ring), 792 (C-S, str.), 838 (para-disustituted aromatic ring), 856 (aromatic $\mathrm{C}=\mathrm{C}$ ), 967 (aromatic $=\mathrm{C}-\mathrm{H}$, bend), 1092 (sym. C-O-C, str.), 1145 (aromatic C-O, str.), 1199 (=C-N), 1248 (asym. C-O-C, str.), 1381 (C-N), 1545 (-NH, bend), $1751(>\mathrm{C}=\mathrm{O}), 3254(-\mathrm{NH})$.

$N$-[2-(3,4-dimethoxyphenyl)-4-oxo-1,3-thiazolidin-3-yl]-2-(3-methyl-2-

oxoquinoxalin-1(2H)-yl)acetamide (5f): Brown solid, yield $65 \%, \mathrm{R}_{f(\mathrm{TEF})} 0.72$, m.p. $173^{\circ} \mathrm{C}$, ${ }^{1} \mathrm{H}-\mathrm{NMR}$ (DMSO- $\left.d_{6}, 300 \mathrm{MHz}, \delta \mathrm{ppm}\right): 8.118(\mathrm{~s}, 1 \mathrm{H}, \mathrm{NHCO}), 7.718-7.788(\mathrm{~m}, 4 \mathrm{H}$, quinoxaline ring protons, $\mathrm{J}=1.8-7.5 \mathrm{~Hz}), 7.410-7.432(\mathrm{~d}, 1 \mathrm{H}, \mathrm{Ar}-\mathrm{H}, \mathrm{J}=6.6 \mathrm{~Hz}), 7.465-$ 7.487 (d, 1H, Ar-H, , J = 6.6 Hz), 7.372-7.396 (d, 1H, Ar-H, J = 7.2 Hz), $5.413\left(\mathrm{~s}, 2 \mathrm{H}, \mathrm{CH}_{2}\right)$, $2.301\left(\mathrm{~s}, 3 \mathrm{H}, \mathrm{CH}_{3}\right), 3.749$ (s, 1H, CH-Ar), $2.517\left(\mathrm{~s}, 2 \mathrm{H}, \mathrm{CH}_{2}-\mathrm{S}\right), 2.384\left(\mathrm{~s}, 6 \mathrm{H},-\mathrm{OCH}_{3}\right)$; IR $\left(\mathrm{KBr}, \mathrm{cm}^{-1}\right)$ : 762 (ortho-disubstituted aromatic ring), $790(\mathrm{C}-\mathrm{S}, \mathrm{str}$.), 842 (para-disustituted aromatic ring), 858 (aromatic $\mathrm{C}=\mathrm{C}$ ), 965 (aromatic $=\mathrm{C}-\mathrm{H}$, bend), 1088 (sym. C-O-C, str.), 1148 (aromatic C-O, str.), 1195 (=C-N), 1248 (asym. C-O-C, str.), 1380 (C-N), 1549 (-NH, bend), $1753(>\mathrm{C}=\mathrm{O}), 3248(-\mathrm{NH})$.

$\mathrm{N}$-[2-(4-dimethylaminophenyl)-4-oxo-1,3-thiazolidin-3-yl]-2-(3-methyl-2oxoquinoxalin-1(2H)-yl)acetamide (5g): Brown solid, yield $64 \%, \mathrm{R}_{f(\mathrm{TEF})} 0.82$, m.p. $205^{\circ} \mathrm{C}$, ${ }^{1} \mathrm{H}-\mathrm{NMR}$ (DMSO- $\left.d_{6}, 300 \mathrm{MHz}, \delta \mathrm{ppm}\right): 8.119$ (s, 1H, NHCO), 7.719-7.801 (m, 4H, quinoxaline ring protons, $\mathrm{J}=1.8-7.5 \mathrm{~Hz}$ ), 7.424-7.482 (q, $4 \mathrm{H}, \mathrm{A}_{2} \mathrm{~B}_{2}$ parrten, $\mathrm{Ar}-\mathrm{H}, \mathrm{J}=3-7.2$ $\mathrm{Hz}), 5.408$ (s, 2H, $\left.\mathrm{CH}_{2}\right), 2.296\left(\mathrm{~s}, 3 \mathrm{H}, \mathrm{CH}_{3}\right), 3.745$ (s, 1H, CH-Ar), 2.515 (s, 2H, CH $\mathrm{CH}_{2} \mathrm{~S}$ ), $2.374\left(\mathrm{~s}, 6 \mathrm{H},-\mathrm{N}\left(\mathrm{CH}_{3}\right)_{2}\right)$; IR $\left(\mathrm{KBr}, \mathrm{cm}^{-1}\right)$ : 766 (ortho-disubstituted aromatic ring), 791 (C-S, str.), 840 (para-disustituted aromatic ring), 862 (aromatic $\mathrm{C}=\mathrm{C}$ ), 965 (aromatic $=\mathrm{C}-\mathrm{H}$, bend), $1198(=\mathrm{C}-\mathrm{N}), 1385(\mathrm{C}-\mathrm{N}), 1552$ (-NH, bend), $1748(>\mathrm{C}=\mathrm{O}), \mathbf{3 2 5 0}(-\mathrm{NH})$.

2-(3-methyl-2-oxoquinoxalin-1(2H)-yl)- $\mathrm{N}$-\{4-oxo-2-[4-(trifluoromethyl)phenyl]1,3-thiazolidin-3-yl \}acetamide (5h): Brown solid, yield 68\%, $\mathrm{R}_{f(\mathrm{TEF})} 0.83$, m.p. $235^{\circ} \mathrm{C},{ }^{1} \mathrm{H}$ NMR (DMSO- $\left.d_{6}, 300 \mathrm{MHz}, \delta \mathrm{ppm}\right): 8.121$ (s, 1H, NHCO), 7.719-7.888 (m, 4H, quinoxaline ring protons, $\mathrm{J}=1.8-7.2 \mathrm{~Hz}), 7.512-7.466\left(\mathrm{q}, 4 \mathrm{H}, \mathrm{A}_{2} \mathrm{~B}_{2}\right.$ parrten, $\mathrm{Ar}-\mathrm{H}, \mathrm{J}=3-6.6$ $\mathrm{Hz}), 5.416\left(\mathrm{~s}, 2 \mathrm{H}, \mathrm{CH}_{2}\right), 2.302\left(\mathrm{~s}, 3 \mathrm{H}, \mathrm{CH}_{3}\right), 3.761$ (s, $\left.1 \mathrm{H}, \mathrm{CH}-\mathrm{Ar}\right), 2.518$ (s, 2H, $\left.\mathrm{CH}_{2}-\mathrm{S}\right)$; IR 
$\left(\mathrm{KBr}, \mathrm{cm}^{-1}\right): 768$ (ortho-disubstituted aromatic ring), 792 (C-S, str.), 845 (para-disustituted aromatic ring), 861 (aromatic $\mathrm{C}=\mathrm{C}), 962$ (aromatic $=\mathrm{C}-\mathrm{H}$, bend), $1188(=\mathrm{C}-\mathrm{N}), 1287$ (aryl C-F, str.), 1392 (C-N), 1565 (-NH, bend), $1752(>\mathrm{C}=\mathrm{O}), 3248(-\mathrm{NH})$.

$\mathrm{N}$-[2-(2-hydroxyphenyl)-4-oxo-1,3-thiazolidin-3-yl]-2-(3-methyl-2-oxoquinoxalin1(2H)-yl)acetamide (5i): Light brown solid, yield $58 \%$, $\mathrm{R}_{f(\mathrm{TEF})} 0.74$, m.p. $195^{\circ} \mathrm{C},{ }^{1} \mathrm{H}-\mathrm{NMR}$ $\left(\mathrm{DMSO}_{6}, 300 \mathrm{MHz}, \delta \mathrm{ppm}\right): 8.120(\mathrm{~s}, 1 \mathrm{H}, \mathrm{NHCO}), 7.715-7.792(\mathrm{~m}, 4 \mathrm{H}$, quinoxaline ring protons, $\mathrm{J}=1.5-7.8 \mathrm{~Hz}$ ), 7.315-7.344 (dd, 2H, Ar-H), 7.409-7.445 (d, 1H, Ar-H, J = 7.8 Hz), 7.328-7.363 (t, 1H, Ar-H, J = 3.3-7.2 Hz), $5.411\left(\mathrm{~s}, 2 \mathrm{H}, \mathrm{CH}_{2}\right), 2.291\left(\mathrm{~s}, 3 \mathrm{H}, \mathrm{CH}_{3}\right), 3.738$ (s, $1 \mathrm{H}, \mathrm{CH}-\mathrm{Ar}), 2.518$ (s, 2H, $\left.\mathrm{CH}_{2}-\mathrm{S}\right), 2.824(\mathrm{~s}, 1 \mathrm{H}$, phenolic $-\mathrm{OH})$; IR $\left(\mathrm{KBr}, \mathrm{cm}^{-1}\right): 766$ (orthodisubstituted aromatic ring), $792(\mathrm{C}-\mathrm{S}$, str.), 864 (aromatic $\mathrm{C}=\mathrm{C}$ ), 963 (aromatic $=\mathrm{C}-\mathrm{H}$, bend), $1199(=\mathrm{C}-\mathrm{N}), 1381(\mathrm{C}-\mathrm{N}), 1550(-\mathrm{NH}$, bend), $1752(>\mathrm{C}=\mathrm{O}), 3255(-\mathrm{NH}), 3567$ (phenolic -OH).

$\mathrm{N}$-[2-(3-Hydroxy-4-methoxyphenyl)-4-oxo-1,3-thiazolidin-3-yl]-2-(3-methyl-2oxoquinoxalin-1(2H)-yl)acetamide (5j): Light brown solid, yield $70 \%, \mathrm{R}_{f(\mathrm{TEF})} 0.78$, m.p. $285^{\circ} \mathrm{C},{ }^{1} \mathrm{H}-\mathrm{NMR}$ (DMSO- $\left.d_{6}, 300 \mathrm{MHz}, \delta \mathrm{ppm}\right): 8.118(\mathrm{~s}, 1 \mathrm{H}, \mathrm{NHCO}), 7.715-7.798(\mathrm{~m}, 4 \mathrm{H}$, quinoxaline ring protons, $\mathrm{J}=1.8-8.1 \mathrm{~Hz}), 7.311-7.337(\mathrm{t}, 1 \mathrm{H}, \mathrm{Ar}-\mathrm{H}, \mathrm{J}=1.8-6 \mathrm{~Hz}), 7.410(\mathrm{~s}$, $1 \mathrm{H}, \mathrm{Ar}-\mathrm{H}), 7.377-7.398(\mathrm{~d}, 1 \mathrm{H}, \mathrm{Ar}-\mathrm{H}, \mathrm{J}=6.3 \mathrm{~Hz}), 5.418\left(\mathrm{~s}, 2 \mathrm{H}, \mathrm{CH}_{2}\right), 2.288\left(\mathrm{~s}, 3 \mathrm{H}, \mathrm{CH}_{3}\right)$, 3.741 (s, 1H, CH-Ar), 2.519 (s, 2H, $\left.\mathrm{CH}_{2}-\mathrm{S}\right), 2.817$ (s, 1H, phenolic - $\left.\mathrm{OH}\right), 3.311(\mathrm{~s}, 3 \mathrm{H},-$ $\mathrm{OCH}_{3}$ ); IR $\left(\mathrm{KBr}, \mathrm{cm}^{-1}\right)$ : 764 (ortho-disubstituted aromatic ring), 789 (C-S, str.), 865 (aromatic $\mathrm{C}=\mathrm{C}), 964$ (aromatic $=\mathrm{C}-\mathrm{H}$, bend), $1201(=\mathrm{C}-\mathrm{N}), 1285(\mathrm{C}-\mathrm{O}$, str. coupled with $\mathrm{H}-$ $\mathrm{C}-\mathrm{H}), 1385$ (C-N), 1552 (-NH, bend), 1752 (>C=O), 3251 (-NH), 3542 (phenolic -OH).

$\mathrm{N}$-[2-(2-fluorophenyl)-4-oxo-1,3-thiazolidin-3-yl]-2-(3-methyl-2-oxoquinoxalin-

1(2H)-yl)acetamide (5k): Dark brown solid, yield $50 \%$, $\mathrm{R}_{f(\mathrm{TEF})} 0.76$, m.p. $235^{\circ} \mathrm{C},{ }^{1} \mathrm{H}-\mathrm{NMR}$ $\left(\right.$ DMSO- $\left.d_{6}, 300 \mathrm{MHz}, \delta \mathrm{ppm}\right): 8.132(\mathrm{~s}, 1 \mathrm{H}, \mathrm{NHCO}), 7.715-7.812(\mathrm{~m}, 4 \mathrm{H}$, quinoxaline ring protons, $\mathrm{J}=1.2-8.1 \mathrm{~Hz}), 7.322-7.381(\mathrm{dd}, 2 \mathrm{H}, \mathrm{Ar}-\mathrm{H}), 7.411-7.446(\mathrm{~d}, 1 \mathrm{H}, \mathrm{Ar}-\mathrm{H}, \mathrm{J}=10.5$ $\mathrm{Hz}), 7.569-7.602(\mathrm{t}, 1 \mathrm{H}, \mathrm{Ar}-\mathrm{H}, \mathrm{J}=3.3-6.6 \mathrm{~Hz}), 5.418\left(\mathrm{~s}, 2 \mathrm{H}, \mathrm{CH}_{2}\right), 2.288\left(\mathrm{~s}, 3 \mathrm{H}, \mathrm{CH}_{3}\right)$, $3.745(\mathrm{~s}, 1 \mathrm{H}, \mathrm{CH}-\mathrm{Ar}), 2.522\left(\mathrm{~s}, 2 \mathrm{H}, \mathrm{CH}_{2}-\mathrm{S}\right)$; IR $\left(\mathrm{KBr}, \mathrm{cm}^{-1}\right): 768$ (ortho-disubstituted aromatic ring), 795 (C-S, str.), 868 (aromatic $\mathrm{C}=\mathrm{C}$ ), 962 (aromatic $=\mathrm{C}-\mathrm{H}$, bend), 1199 (=CN), 1285 (aryl C-F, str.), 1385 (C-N), 1541 (-NH, bend), 1747 (>C=O), 3199 (-NH).

$\mathrm{N}$-[2-(2-chlorophenyl)-4-oxo-1,3-thiazolidin-3-yl]-2-(3-methyl-2-oxoquinoxalin1(2H)-yl)acetamide (5l): Light brown solid, yield $52 \%$, $\mathrm{R}_{f}$ (TEF) 0.78 , m.p. $225^{\circ} \mathrm{C},{ }^{1} \mathrm{H}-\mathrm{NMR}$ $\left(\right.$ DMSO- $\left.d_{6}, 300 \mathrm{MHz}, \delta \mathrm{ppm}\right): 8.133(\mathrm{~s}, 1 \mathrm{H}, \mathrm{NHCO}), 7.710-7.798(\mathrm{~m}, 4 \mathrm{H}$, quinoxaline ring protons, $\mathrm{J}=1.2-8.1 \mathrm{~Hz}$ ), 7.318-7.345 (dd, 2H, Ar-H), 7.408-7.435 (d, 1H, Ar-H, J = 8.1 Hz), 7.355-7.389 (t, 1H, Ar-H, J = 3-7.2 Hz), $5.412\left(\mathrm{~s}, 2 \mathrm{H}, \mathrm{CH}_{2}\right), 2.287\left(\mathrm{~s}, 3 \mathrm{H}, \mathrm{CH}_{3}\right), 3.735$ (s, $1 \mathrm{H}, \mathrm{CH}-\mathrm{Ar}$ ), 2.525 (s, $\left.2 \mathrm{H}, \mathrm{CH}_{2}-\mathrm{S}\right)$; IR $\left(\mathrm{KBr}, \mathrm{cm}^{-1}\right)$ : 765 (ortho-disubstituted aromatic ring), 793 (C-S, str.), 872 (aromatic C=C), 965 (aromatic $=\mathrm{C}-\mathrm{H}$, bend), 1132 (aryl C-Cl, str.), 1201 $(=\mathrm{C}-\mathrm{N}), 1286$ (aryl C-F, str.), 1383 (C-N), 1541 (-NH, bend), 1747 (>C=O), $3199(-\mathrm{NH})$.

\section{Antibacterial \& Antifungal Activity}

All the synthesized have been screened for in-vitro antibacterial activity against gram positive bacteria $S$. aureus (ATCC2913), gram negative bacteria $K$. pneumonia (ATCC700603), P. aeruginosa (ATCC27853), E. coli (ATCC25922) and antifungal activity against C. albicans (MTCC3017), A. niger (MTCC281) and A. flavus (MTCC277) by using cup-plate method.

The growth media (Nutrient Agar media for bacterial growth \& Sabouraud Dextrose Agar media for fungal growth) were prepared \& sterilized in autoclave at 15 psig for 15 minutes. These media were poured into petri-plates under standard conditions \& allowed to solidify. On the surface of media, test microorganisms were inoculated with sterilized nickel loop wire. Cups were made by boring on the surface of growth media with previously sterilized borer. Four cups were made on each petri-plate. These cups were filled with 
different concentrations (50 $\mu \mathrm{g} / \mathrm{mL} \& 100 \mu \mathrm{g} / \mathrm{mL}$ in DMSO) of the test compounds, third with control (DMSO) \& fourth one with standard drug. The plates were kept in cold for 1 hour to allow the diffusion of test compounds \& then incubated at $35^{\circ} \mathrm{C}$ for 48 hours (for antifungal activity) \& at $37^{\circ} \mathrm{C}$ for 24 hours (for antibacterial activity). The zones of inhibition formed around the cups after respective incubation were measured.

\section{Results and Discussion}

All the newly synthesized compounds have been evaluated for their antibacterial activity against $E$. coli, $S$. aureus, $K$. pneumoniae, $P$. aeruginosa and antifungal activity against $C$. albicans, A. niger and A. flavus. In the present communication, we have concentrated on "the effect of various substituents on phenyl ring attached to 2-position of thiazolidinone nucleus attached to $\mathrm{N}$-atom of acetamido functionality on 1-position of 3-methyl-2-oxoquinoxaline -1 $(2 H)$-acetamide". Against E.coli, compounds with functionalities 4-Cl (5b), 4$\mathrm{OH}(\mathbf{5 c}), 4-\mathrm{NO}_{2}(\mathbf{5 d}), 4-\mathrm{N}\left(\mathrm{CH}_{3}\right)_{2}(\mathbf{5 g}), 4-\mathrm{CF}_{3}(\mathbf{5 h}), 2-\mathrm{F}(\mathbf{5 k})$ on phenyl ring attached to 2position of thiazolidinone nucleus attached to above mentioned position in basic scaffold have exhibited maximal activity. Compound 5i having $-\mathrm{OH}$ group at ortho-position on phenyl group at above mentioned position exhibited minimal activity. Thiazolidinone nucleus with electron withdrawing functional groups 4-Cl (5b), 4-OH (5c), 4- $\mathrm{NO}_{2}(\mathbf{5 d}), 4-$ $\mathrm{CF}_{3}$ (5h), 3-OH-4-OCH $\mathrm{OCH}_{3}(\mathbf{5 j})$ and 2-F (5k) have shown good activity against $S$. aureus. However, -OH group at ortho-position of phenyl ring (5i) on 2-position of thiazolidinone ring exhibited minimal activity against $S$. aureus. Compounds with electron donating groups 4- $\mathrm{OCH}_{3}(\mathbf{5 e}), 3,4-\left(\mathrm{OCH}_{3}\right)_{2}$ (5f) exhibited moderate activity against $S$. aureus. Against $K$. pneumoniae, compounds with functional groups 4-Cl (5b), 4-OH (5c), 4- $\mathrm{NO}_{2}(\mathbf{5 d}), 4-$ $\left.\mathrm{N}\left(\mathrm{CH}_{3}\right)_{2} \mathbf{( 5 g}\right), 4-\mathrm{CF}_{3}(\mathbf{5 h})$ on phenyl ring attached to 2-position of thiazolidinone ring exhibited fantastic activity. Additionally, among thiazolidinone derivatives, compounds with 4- $\mathrm{OCH}_{3}(\mathbf{5 e}), 3-\mathrm{OH}-4-\mathrm{OCH}_{3}(\mathbf{5 j})$ on above mentioned position also exhibited better activity. Compounds $5 \mathbf{f}$ and $5 \mathbf{l}$ having 3,4- $\left(\mathrm{OCH}_{3}\right)_{2}-\mathrm{C}_{6} \mathrm{H}_{3}$ and 2-Cl- $\mathrm{C}_{6} \mathrm{H}_{4}$ on 2-position of thiazolidinone ring respectively have shown minimal activity against $K$. pneumoniae. Most of newly synthesized compounds have shown good antibacterial activity against $P$. aeruginosa. Compounds having 4-Cl (5b), 4-OH (5c), 4- $\mathrm{NO}_{2}(\mathbf{5 d}), 4-\mathrm{N}\left(\mathrm{CH}_{3}\right)_{2}(\mathbf{5 g}), 4-\mathrm{CF}_{3}$ (5h), and 3-OH-4-OCH $\mathrm{OCH}_{3}(\mathbf{5 j})$ on phenyl ring attached to above mentioned positions at thiazolidinone ring exhibited better activity. Thiazolidinone derivatives with $3,4-\left(\mathrm{OCH}_{3}\right)_{2}-$ $\mathrm{C}_{6} \mathrm{H}_{3}$ (5f) and 2-Cl- $\mathrm{C}_{6} \mathrm{H}_{4}$ (5l) exhibited minimal activity.

Against fungal strains $C$. albicans, A. niger, A. flavus, most of the newly synthesized compound exhibited moderate activity. Against $C$. albicans compounds $5 \mathbf{5 a}, \mathbf{5 b}, \mathbf{5 c}, \mathbf{5 d}, \mathbf{5 g}$ and $\mathbf{5} \boldsymbol{h}$ having unsubstituted phenyl ring, 4-Cl- $\left.\mathrm{C}_{6} \mathrm{H}_{4}, 4-\mathrm{OH}-\mathrm{C}_{6} \mathrm{H}_{4}, 4-\mathrm{NO}_{2}-\mathrm{C}_{6} \mathrm{H}_{4}, 4-\mathrm{N}_{(} \mathrm{CH}_{3}\right)_{2}-$ $\mathrm{C}_{6} \mathrm{H}_{4}$ and $4-\mathrm{CF}_{3}-\mathrm{C}_{6} \mathrm{H}_{4}$ groups in respective position exhibited fantastic activity. However, compound $\mathbf{5 k}$ and $\mathbf{5 l}$ having halogens $\mathrm{F}$ and $\mathrm{Cl}$ on ortho-positions on phenyl ring attached to 2-position of thiazolidinone ring exhibited minimal activity. Against, A. niger, thiazolidinone derivatives, $\mathbf{5 d}, \mathbf{5} \mathbf{h}$ and $\mathbf{5} \mathbf{j}$ having $4-\mathrm{NO}_{2}, 4-\mathrm{CF}_{3}$ and $3-\mathrm{OH}-4-\mathrm{OCH}_{3}$ groups on phenyl ring were found to be more active. Thiazolidinone derivatives, $\mathbf{5 i}$, $\mathbf{5} \mathbf{k}$ and $\mathbf{5 l}$ having 2-OH, 2-F and 2-Cl on phenyl ring attached to 2-position of thiazolidinone ring exhibited minimal activity. Compounds with $4-\mathrm{Cl}_{-} \mathrm{C}_{6} \mathrm{H}_{4}(\mathbf{5 b}), 4-\mathrm{OH}-\mathrm{C}_{6} \mathrm{H}_{4}(\mathbf{5 c}), 4-\mathrm{NO}_{2}-\mathrm{C}_{6} \mathrm{H}_{4}$ (5d) and 4- $\mathrm{CF}_{3}-\mathrm{C}_{6} \mathrm{H}_{4}$ (5h), have shown better activity, while compounds with 3,4- $\left(\mathrm{OCH}_{3}\right)_{2}-\mathrm{C}_{6} \mathrm{H}_{3}(\mathbf{5 f})$, $2-\mathrm{OH}-\mathrm{C}_{6} \mathrm{H}_{4}$ (5i), 2-F- $\mathrm{C}_{6} \mathrm{H}_{4}(\mathbf{5 k})$ and $2-\mathrm{Cl}-\mathrm{C}_{6} \mathrm{H}_{4}$ (5l) were reported as minimal active compounds. Against $A$. flavus, among thiazolidinone derivatives, $\mathbf{5 b}, \mathbf{5 c}, \mathbf{5 d}, \mathbf{5 g}$ and $\mathbf{5 h}$ with 4-Cl, 4-OH, 4-NO $\mathrm{N}_{2}, 4-\mathrm{N}\left(\mathrm{CH}_{3}\right)_{2}$ and $4-\mathrm{CF}_{3}$ substitutions on phenyl ring attached to $\mathrm{N}$-atom of acetamido group on first position of 3-methyl-2-oxo-quinoxaline-1 $(2 H)$-acetamide exhibited fantastic activity against all bacterial strains under investigation. 
Table 1. Zone of inhibition (mm) of different compounds.

\begin{tabular}{|c|c|c|c|c|c|c|c|c|c|c|c|c|c|c|}
\hline \multirow{2}{*}{ 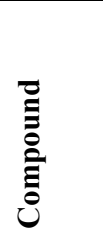 } & \multicolumn{2}{|c|}{ E. coli } & \multicolumn{2}{|c|}{ S. aureus } & \multicolumn{2}{|c|}{$\begin{array}{c}\text { K. } \\
\text { pneumoni } \\
\text { ae }\end{array}$} & \multicolumn{2}{|c|}{$\begin{array}{c}P . \\
\text { aeruginos } \\
a\end{array}$} & \multicolumn{2}{|c|}{$\begin{array}{c}C . \\
\text { albicans }\end{array}$} & \multicolumn{2}{|c|}{ A. niger } & \multicolumn{2}{|c|}{ A. flavus } \\
\hline & $\begin{array}{c}50 \\
\mu \mathrm{g} / \\
\mathrm{ml}\end{array}$ & $\begin{array}{c}100 \\
\mu \mathrm{g} / \\
\mathrm{ml}\end{array}$ & $\begin{array}{c}50 \\
\mu \mathrm{g} / \\
\mathrm{ml}\end{array}$ & $\begin{array}{c}100 \\
\mu \mathrm{g} / \\
\mathrm{ml}\end{array}$ & $\begin{array}{c}50 \\
\mu \mathrm{g} / \\
\mathrm{ml}\end{array}$ & $\begin{array}{c}100 \\
\mu \mathrm{g} / \\
\mathrm{ml}\end{array}$ & $\begin{array}{c}50 \\
\mu \mathrm{g} / \\
\mathrm{ml}\end{array}$ & $\begin{array}{c}100 \\
\mu \mathrm{g} / \\
\mathrm{ml}\end{array}$ & $\begin{array}{c}50 \\
\mu \mathrm{g} / \\
\mathrm{ml}\end{array}$ & $\begin{array}{c}100 \\
\mu \mathrm{g} / \\
\mathrm{ml}\end{array}$ & $\begin{array}{c}50 \\
\mu \mathrm{g} / \\
\mathrm{ml}\end{array}$ & $\begin{array}{c}100 \\
\mu \mathrm{g} / \\
\mathrm{ml}\end{array}$ & $\begin{array}{c}50 \\
\mu \mathrm{g} / \\
\mathrm{ml}\end{array}$ & $\begin{array}{c}100 \\
\mu \mathrm{g} / \\
\mathrm{ml}\end{array}$ \\
\hline $5 a$ & 11 & 18 & 10 & 17 & 10 & 16 & 11 & 20 & 12 & 22 & 11 & 19 & 11 & 20 \\
\hline 6b & 13 & 22 & 13 & 21 & 14 & 24 & 14 & 25 & 12 & 21 & 11 & 19 & 13 & 23 \\
\hline $6 c$ & 12 & 21 & 13 & 23 & 14 & 25 & 14 & 24 & 13 & 22 & 11 & 20 & 12 & 21 \\
\hline 6d & 13 & 23 & 13 & 23 & 13 & 22 & 13 & 23 & 12 & 21 & 13 & 23 & 13 & 20 \\
\hline $6 e$ & 09 & 16 & 11 & 19 & 12 & 21 & 11 & 19 & 11 & 20 & 09 & 14 & 09 & 15 \\
\hline $6 f$ & 10 & 17 & 09 & 16 & 08 & 14 & 09 & 14 & 10 & 18 & 10 & 17 & 08 & 13 \\
\hline $6 g$ & 13 & 22 & 12 & 21 & 13 & 23 & 14 & 24 & 14 & 24 & 11 & 18 & 10 & 17 \\
\hline $6 h$ & 14 & 25 & 14 & 24 & 14 & 24 & 14 & 25 & 14 & 24 & 13 & 22 & 14 & 24 \\
\hline $6 \mathbf{i}$ & 07 & 12 & 07 & 11 & 09 & 15 & 08 & 13 & 09 & 14 & 08 & 13 & 08 & 14 \\
\hline $6 j$ & 11 & 18 & 13 & 22 & 12 & 21 & 12 & 22 & 11 & 20 & 12 & 21 & 10 & 18 \\
\hline $6 k$ & 12 & 22 & 11 & 20 & 11 & 19 & 11 & 18 & 08 & 14 & 09 & 15 & 08 & 14 \\
\hline 61 & 09 & 16 & 09 & 15 & 07 & 12 & 08 & 14 & 07 & 12 & 07 & 11 & 08 & 14 \\
\hline $\begin{array}{c}\text { Stand } \\
\text { ard }\end{array}$ & \multicolumn{2}{|c|}{27} & \multicolumn{2}{|c|}{$\frac{1}{28}$} & \multicolumn{2}{|c|}{27} & \multicolumn{2}{|c|}{27} & \multicolumn{2}{|c|}{26} & \multicolumn{2}{|c|}{26} & \multicolumn{2}{|c|}{25} \\
\hline $\begin{array}{c}\text { Contr } \\
\text { ol }\end{array}$ & \multicolumn{2}{|c|}{02} & \multicolumn{2}{|c|}{02} & \multicolumn{2}{|c|}{02} & \multicolumn{2}{|c|}{02} & \multicolumn{2}{|c|}{02} & \multicolumn{2}{|c|}{03} & \multicolumn{2}{|c|}{02} \\
\hline
\end{tabular}

Standard Drugs:

For antibacterial activity: Ciprofloxacin, $30 \mu \mathrm{g} / \mathrm{ml}$.

For antifungal activity: Voriconazole, $30 \mu \mathrm{g} / \mathrm{ml}$.

Control: DMSO.

Compounds $\mathbf{5 d}$ and $\mathbf{5 h}$ were found to be more active against all fungal strains under investigation. Compounds $\mathbf{5 d}$ and $\mathbf{5 h}$ were also found to be more active against all bacterial and fungal strains under investigation. Compound $\mathbf{5 l}$ having 2-Cl group on phenyl ring attached to 4-position of thiazolidinone ring showed minimal activity against all bacterial and fungal strains.

\section{Conclusion}

All the synthesized compounds were evaluated for their antibacterial activity against $E$. coli, $S$. aureus, $P$. aeruginosa, $K$. pneumoniae and antifungal activity against $C$. albicans, $A$. niger and $A$. flavus using cup-plate method. 2-(3-Methyl-2-oxoquinoxalin-1(2H)yl)acetamide based thiazolidinone derivatives having aromatic phenyl ring substituted with 4-Cl, 4-OH, 4- $\mathrm{NO}_{2}, 4-\mathrm{N}\left(\mathrm{CH}_{3}\right)_{2}$ and 4- $\mathrm{CF}_{3}$ exhibited fantastic activity against all the bacterial strains under investigation. 2-(3-Methyl-2-oxoquinoxalin-1 $(2 \mathrm{H})$-yl)acetamide based thiazolidinone derivatives having aromatic phenyl ring substituted with $4-\mathrm{NO}_{2}$ and $4-\mathrm{CF}_{3}$ have exhibited better activity against all bacteria and fungi under investigation. These compounds may act as lead for future investigation. 


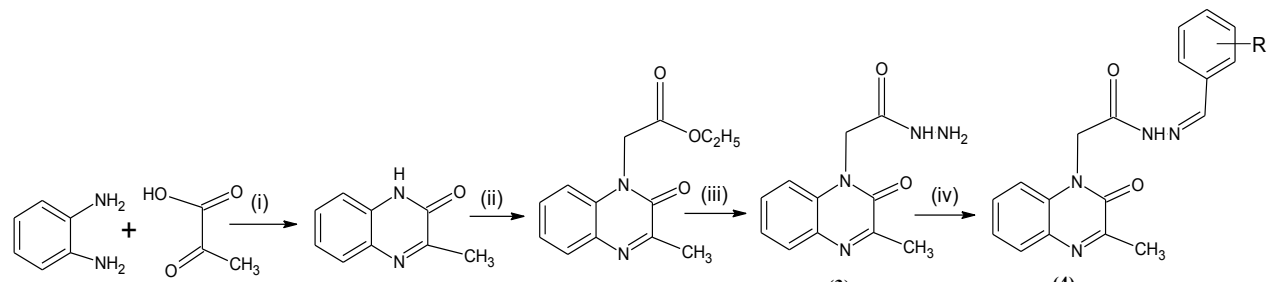

(1)

(2)

(3)

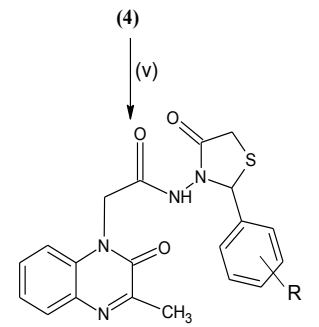

(5)

\begin{tabular}{|c|l|l|l|l|l|l|l|l|l|l|l|l|}
\hline Compound & $\mathbf{4 a}, \mathbf{5 a}$ & $\mathbf{4 b}, \mathbf{5 b}$ & $\mathbf{4 c}, \mathbf{5 c}$ & $\mathbf{4 d}, \mathbf{5 d}$ & $\mathbf{4 e}, \mathbf{5 e}$ & $\mathbf{4 f}, \mathbf{5 f}$ & $\mathbf{4 g}, \mathbf{5 g}$ & $\mathbf{4 h}, \mathbf{5 h}$ & $\mathbf{4 i}, \mathbf{5 i}$ & $\mathbf{4 j}, \mathbf{5 j}$ & $\mathbf{4 k}, \mathbf{5 k}$ & $\mathbf{4 1}, \mathbf{5} \mathbf{~}$ \\
\hline $\mathbf{R}$ & $\mathrm{H}$ & $4-\mathrm{Cl}$ & $4-\mathrm{OH}$ & $4-\mathrm{NO}_{2}$ & $4-\mathrm{OMe}$ & $3,4-(\mathrm{OMe})_{2}$ & $4-\mathrm{NMe}_{2}$ & $4-\mathrm{CF}_{3}$ & $2-\mathrm{OH}$ & $3-\mathrm{OH}-4-\mathrm{OMe}$ & $2-\mathrm{F}$ & $2-\mathrm{Cl}$ \\
\hline
\end{tabular}

Reagents \& Conditions: (i) $20 \%, \mathrm{HCl}, 45-50^{\circ} \mathrm{C}$, stirring (ii) $\mathrm{ClCH}_{2} \mathrm{COOEt}$, acetone, $40^{\circ} \mathrm{C}$, reflux (iii) $\mathrm{NH}_{2} \mathrm{NH}_{2} \cdot \mathrm{H}_{2} \mathrm{O}, \mathrm{MeOH}$, reflux (iv) $\mathrm{RCHO}$, EtOH, reflux (v) $\mathrm{HSCH}_{2} \mathrm{COOH}$, reflux in heavy petroleum ether bath

Figure 1. Reaction Scheme.

\section{Graphical Representation}<smiles>[R]c1cccc(C2SCC(=O)N2NC(=O)Cn2c(=O)c(C)nc3ccccc32)c1</smiles>

5(a-l)

\section{References}

1. Grande F, Aiello F, Grazia O D, Brizz,i A, Garofalo A, Neamati N, Bioorg. Med. Chem. 2007, 15, 288.

2. Amin K M, Ismail M M F, Noaman E, Soliman D H, Ammar Y A, Bioorg. Med. Chem. 2006, 14, 6917.

3. Zarranz B, Jaso A, Aldana I, Monge A, Bioorg. Med. Chem. 2004, 12, 3711.

4. Lee H, Cho S, Namgoong K, Jung J K, Yang S, Bioorg. Med. Chem. Lett. 2004, 14, 1235.

5. Diana P, Martorana A, Barraja P, Montalbaro A, J. Med. Chem. 2008, 51, 2387.

6. Chung H J, Jung O J, Chae M J, Hong S Y, Chung K H, Lee S K, Bioorg. Med. Chem. Lett. 2005, 15, 3380 . 
7. Katsuyuki A, Obata T, Yamazaki Y, Mori Y, Hirokawa H, Koseki J, Hattori T, Niitsu K, Takeda S, Aburada M, Miyamoto K, Chem. Pharm. Bulletin. 2007, 55, 255.

8. Carta A, Loriga M, Paglietti G, Mattana A, Fiori P L, Mollicotti P, Sechi L, Zanetti S, Eur. J. Med. Chem. 2004, 39, 195.

9. Carta A, Paglietti G, Nikookar M E R, Sanna P, Sechi L, Zanetti S, Eur. J. Med. Chem. 2002, 37, 355.

10. Tandon V K, Yadav D B, Maurya H K, Chaturvedi A K, Shukla P K, Bioorg. Med. Chem. 2006, 14, 6120.

11. Kotharkar S A, Shinde D B, Bioorg. Med. Chem. Lett. 2006, 16, 6181.

12. Mashevskaya I V, Makhmudov R R, Aleksandrova G A, Golovnira O V, Duvalov A V, Maslivets A N, Pharm. Chem. J. 2001, 35, 196.

13. Mashevskaya I V, Tolmacheva I A, Voronova E V, Odegova T F, Alkeksandrova G A, Goleneva A F, Kot'tsova S V, Maslivets A N, Pharm. Chem. J. 2002, 36, 86.

14. Vyas D A, Chauhan N A, Parikh A R, Indian J. Chem. 2007, 46B, 1699.

15. Jaso A, Zarranz B, Aldana I, Monge A, Eur. J. Med. Chem. 2003, 38, 791.

16. Zarranz B, Jaso A, Aldana I, Monge A, Bioorg. Med. Chem. 2003, 11, 2149.

17. Jaso A, Zarranz B, Aldana I, Monge A, J. Med. Chem. 2005, 48, 2019.

18. Seitz L E, Suling W J, Reynolds R C, J. Med. Chem. 2002, 45, 5604.

19. Burguete A, Pontiki E, Litina D H, Villar R, Vicente E, Solano B, Ancizu S, Aldana I, Monge A, Bioorg. Med. Chem. Lett. 2007, 17, 6439.

20. El-Bendary E R, Goda F E, Maaronf A R, Badria F A, Sci. Pharm., 2004, 72, 175.

21. PalekarV S, Damle A J, Shukla S R, Eur. J. Med. Chem. 2009, 44, 5112.

22. Omar K, Geronikaki A, Zoumpoulakis P, Camoutsis C, Soković M, Ćirić A J, Glamočlija J, Bioorg. Med. Chem. 2010, 18, 426.

23. Kumar A, Rajput C S, Bhati S K, Bioorg. Med. Chem. 2007, 15, 3089.

24. Hafez H N, El-Gazzar A R B A, Bioorg. Med. Chem. Lett. 2009, 19, 4143.

25. Ravichandran V, Kumar B R P, Sankar S, Agrawal R K, Eur. J. Med. Chem. 2009, 44, 1180.

26. El-Gaby M S A, El-Hag Ali G A M, El-Maghraby A A, El-Rahman M T A, Helal M H M, Eur. J. Med. Chem. 2009, 44, 4148. 


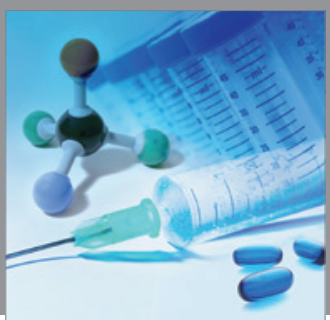

International Journal of

Medicinal Chemistry

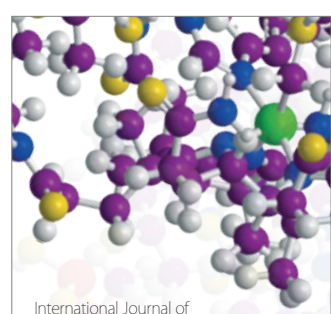

Carbohydrate Chemistry

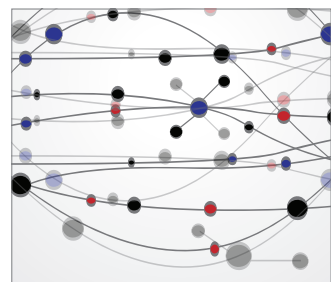

The Scientific World Journal
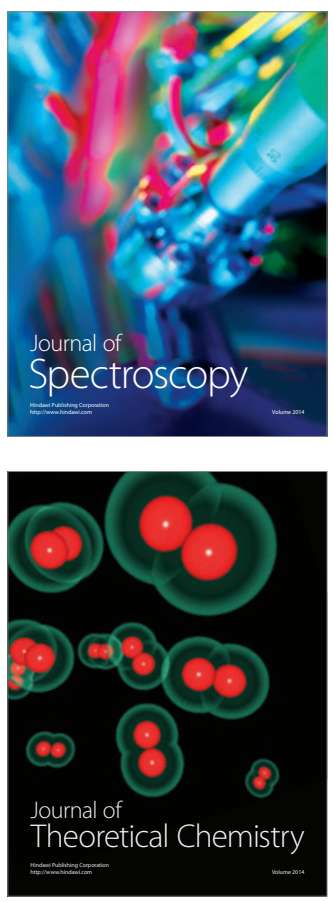
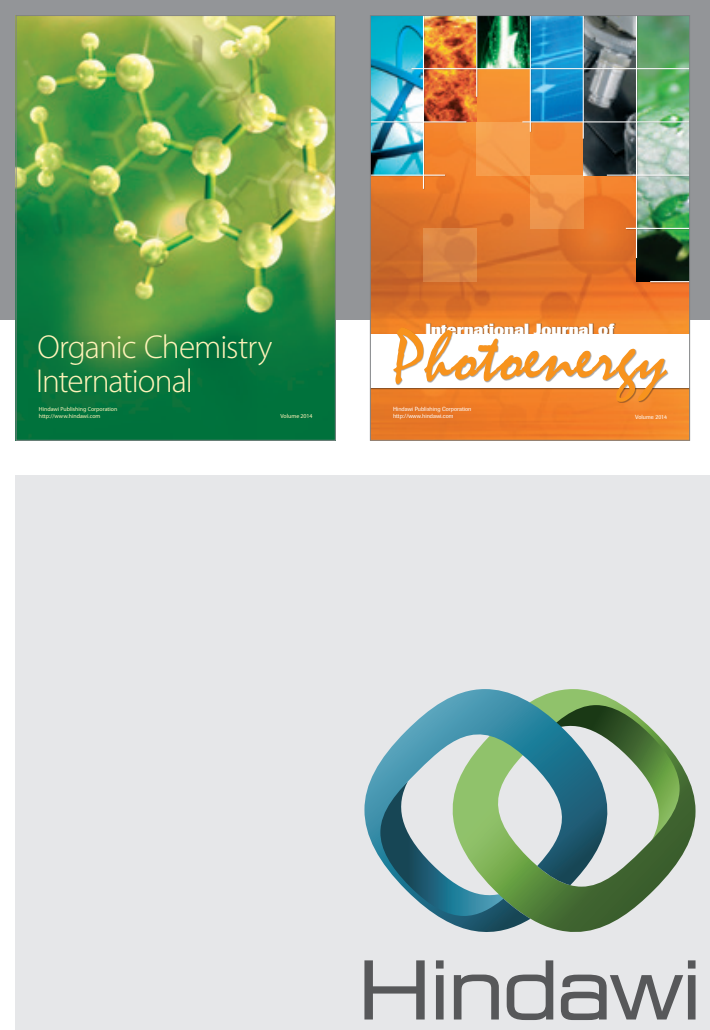

Submit your manuscripts at

http://www.hindawi.com
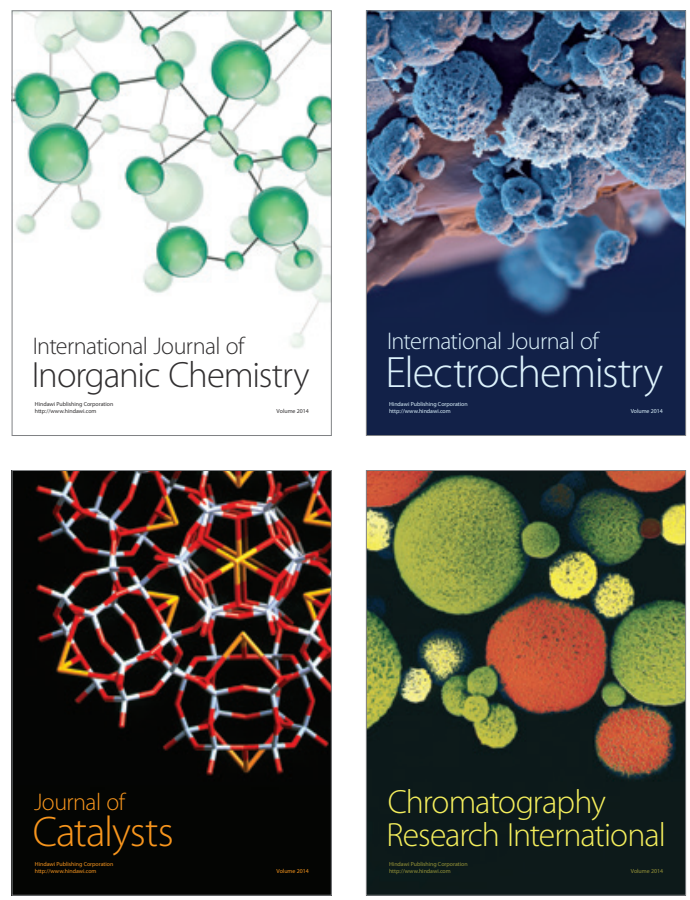
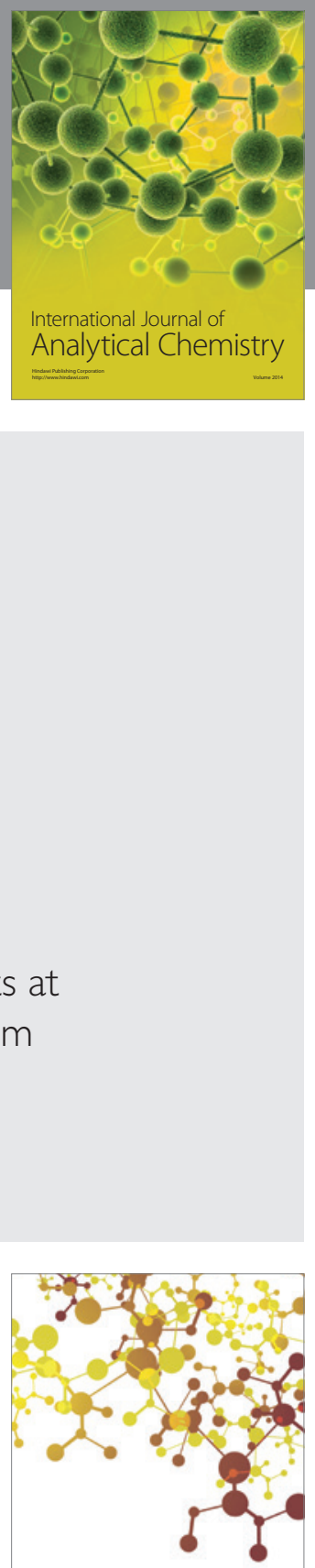

Journal of

Applied Chemistry
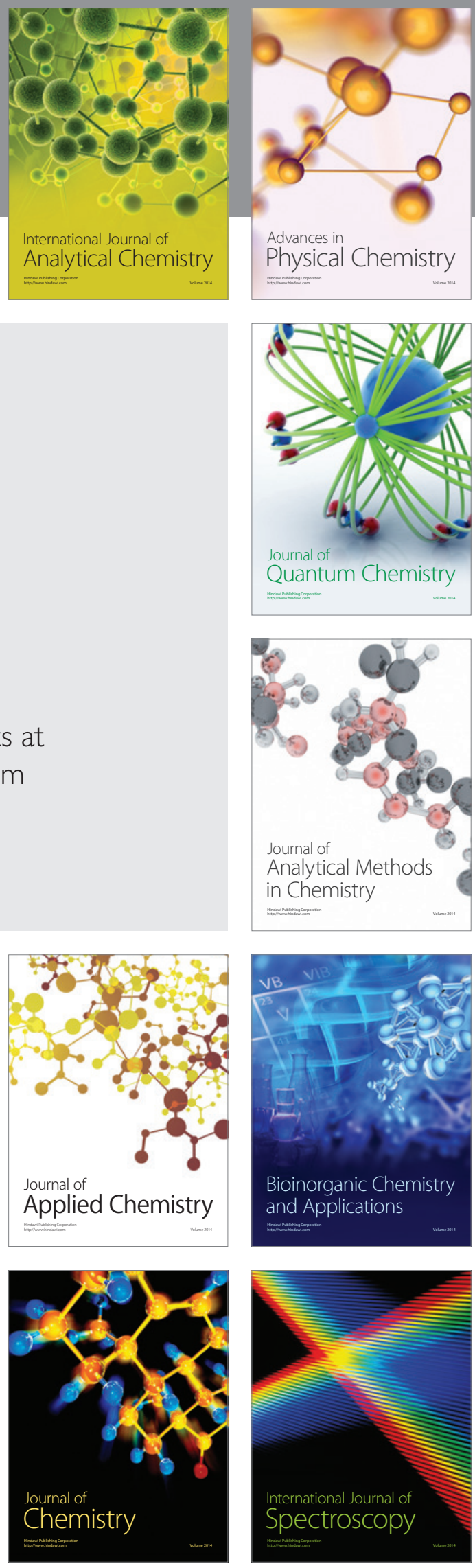